\title{
Philosophiques
}

\section{Alain de Libera, La référence vide. Théories de la proposition, Paris, PUF, coll. "Chaire Étienne Gilson », 2002, 357 pages.}

\section{Frédéric Tremblay}

Volume 32, numéro 1, printemps 2005

Questions d'interprétation

URI : https://id.erudit.org/iderudit/011084ar

DOI : https://doi.org/10.7202/011084ar

Aller au sommaire du numéro

Éditeur(s)

Société de philosophie du Québec

ISSN

0316-2923 (imprimé)

1492-1391 (numérique)

Découvrir la revue

Citer ce compte rendu

Tremblay, F. (2005). Compte rendu de [Alain de Libera, La référence vide. Théories de la proposition, Paris, PUF, coll. " Chaire Étienne Gilson », 2002, 357 pages.] Philosophiques, 32(1), 282-284. https://doi.org/10.7202/011084ar d'utilisation que vous pouvez consulter en ligne.

https://apropos.erudit.org/fr/usagers/politique-dutilisation/ 


\section{Alain de Libera, La référence vide. Théories de la proposition, Paris, PUF, coll. «Chaire Étienne Gilson », 2002, 357 pages.}

En 1993, Perzsyck ${ }^{5}$ regrettait l'absence d'une étude détaillée sur l'histoire de la notion d'objet non existant. Il ignorait alors les travaux d'historiographie de la philosophie médiévale menés en France et entrepris par Hubert Élie lors de la publication, en 1936, de sa thèse sur Grégoire de Rimini et Meinong. La thèse intitulée Le complexe significabile fut réimprimée en 2000 sous le titre Le signifiable par complexe. La proposition et son objet. Grégoire de Rimini, Meinong, Russell. Élie défendait l'idée "que tout ce que Meinong et ses contradicteurs ont dit en la matière, Grégoire de Rimini et ses adversaires l'avaient, à peu de chose près, dit avant eux ${ }^{6}$ ». En 1991, De Libera contribuait à l'histoire commencée par Élie avec un article intitulé «Roger Bacon et la référence vide. Sur quelques antécédents médiévaux du paradoxe de Meinong $^{7}$ ", et il poursuivait en 1997 avec "Subsistance et existence : Porphyre et Meinong $^{8}$ ". C'est en 1999, grâce à Jean-François Courtine, que parut la première traduction française de Meinong.

Le présent livre se situe dans cette lignée qui se donne pour mandat de contribuer au projet contemporain de redessiner la carte des antécédents de la théorie des objets non-existants. Mais l'ambition outrepasse cette histoire pour s'étendre à celle des propositions, complexes signifiables et Sachverhalte («états de choses»). Car si l'objet constitue la proposition ou l'état de choses, comme le juge l'auteur, la proposition elle-même peut être vide. De là le sous-titre, sans doute calqué sur "Theories of the Proposition» de E. J. Ashworth. Enfin, comme le livre aborde le «il y a » et le «es gibt» d'une façon générale, il concourt également à l'histoire de l'ontologie.

Le livre fut constitué à partir de six conférences données en novembre et décembre 2000 lors des réunions de la Chaire Étienne Gilson à l'Institut catholique. Cependant, les titres des chapitres ne correspondent pas avec ceux des conférences, lesquelles furent, selon l'expression de l'auteur, "généreusement modifiées". Voici au moins les titres des chapitres : "Les irréels du passé »; "La constance du sujet »; "Le “Troisième Royaume" "; "Véri-facteurs et véri-porteurs"; "Les faits et les choses"; " "Complexe significabile" et "Sachverhalt" : "l'Argument du Penseur" "; "Tropes ou état de choses?"; "Ce que disent les propositions »; "Envoi».

Adoptant un style analytique en histoire de la philosophie médiévale, l'auteur qualifie son projet d' "archéologie philosophique ", non indifférente à la démarche foucaldienne ${ }^{9}$. S'inspirant de l'approche holiste et relativiste en histoire, Alain de Libera avance que si les vérités ne changeaient pas, elles ne pourraient être reformulées à une époque ultérieure, et l'historien ne pourrait jamais démontrer que telle thèse fut déjà

5. Kenneth J. Perszyk, Nonexistent Objects: Meinong and Contemporary Philosophy, Dordrecht, Kluwer, 1993, p. 68

6. Hubert Élie, Le signifiable par complexe : la proposition et son objet, Grégoire de Rimini, Meinong, Russell, Paris, Vrin, 1936-2000, p. 7-8

7. A. de Libera, "Roger Bacon et la référence vide. Sur quelques antécédents médiévaux du paradoxe de Meinong", Lectionum varietates, Hommage à Paul Vignaux, éd. J. Jolivet, Z. Kluza, Paris, Vrin, 1991, p. 85-120.

8. A. de Libera, "Subsistance et existence : Porphyre et Meinong ", Revue de métaphysique et de morale, 2, 1997, p. 167-192

9. Voir De Libera «Archéologie et reconstruction. Sur la méthode en histoire de la philosophie médiévale» Un siècle de philosophie 1900-2000, Paris, Gallimard, 2000, p. 552-587. 
défendue par tel autre penseur. Ainsi, la méthode consistant à faire l'histoire d'un problème demanderait d'établir une continuité dans l'apparente discontinuité.

Pour démontrer la répétition historique d'une thèse, l'auteur cherche à introduire, dans le domaine de l'histoire de la philosophie de la logique, sa méthode qui consiste à localiser les «complexes de questions et de réponses» (CQR). Les CQR sur le thème de la référence vide $\mathrm{du} \mathrm{XII}^{\mathrm{e}}$ au Xv $\mathrm{Xv}^{\mathrm{e}}$ siècle sont conséquemment comparés, d'abord entre eux, ensuite avec des CQR tirés du $\mathrm{Xx}^{\mathrm{e}}$ siècle. Et puisque les CQR sont "enfouis» (pour utiliser les termes de l'archéologie) dans les sophismata, l'auteur explique le rôle du sophisma au Moyen Âge. Le sophisma n'est pas un sophisme mais une proposition paradoxale du type de celle de Russell ( «l'actuel roi de France est chauve»), couramment utilisée comme coup d'envoi de la disputatio.

Les disputationes d'une vingtaine de scolastiques passent ainsi dans l'appareil. Parmi eux, Porphyre, Avicenne, Abélard, Dun Scott, Gauthier Burley, Jean Buridan, Ockham, Grégoire de Rimini, Henri de Gand et Adam de Wodeham. La plupart des rencontres surviennent entre Oxford et Paris et sont ensuite comparées à des thèses de Bolzano, Brentano, Frege, Meinong, Anton Marty et Adolf Reinach. L'auteur est motivé par la question de la "commensurabilité » entre elles des thèses exprimées par les philosophes.

En parallèle, l'auteur cherche à vérifier sa thèse sur le divorce des courants analytique et continental, repérant dans l'histoire des condamnations et censures de thèses, l'origine du «divorce» entre Oxford et Paris ( "Oxford-Paris split»). Selon l'auteur, on doit le divorce à l'intervention des autorités dans les débats logiques (coutume propre à l'Angleterre) et le véritable split serait advenu au XIV siècle.

La première censure évoquée est celle du chancelier d'Oxford, Robert Kilwardby. Elle interdisait l'enseignement de la thèse selon laquelle «il ne peut y avoir de vérité nécessaire sans constance du sujet». L'interdiction visait la thèse de Roger Bacon voulant que «la nécessité d'une proposition ne s'étend pas au-delà de l'existence du sujet ». Le dessein du chancelier venait de la thèse suivante : "la vérité d'une proposition ne requiert pas en matière naturelle l'existence en acte de ses extrêmes ».

Par conséquent, si Kilwardby avait raison sur Bacon et que la vérité de la proposition est indépendante de son sujet, alors «la pensée n'a pas de porteur », et l'auteur nous conduit par voie de comparaison à la thèse frégéenne d'un "Troisième Royaume ", pour ensuite revenir en arrière, vers Avicenne, selon qui « toute pensée est pensée par Dieu ", thèse à son tour associée au "pays des possibles » de Leibniz, de même qu'à la thèse de Grégoire de Rimini : "avant que le monde ne fût, il existait de nombreux signifiables complexement ". Cela étant, l'auteur poursuit avec une thèse de Henri de Gand sur l'essence considérée comme quelque chose d' "indifférent à l'être et au non-être », qu'il rapproche de la thèse fondamentale de Meinong : «il y a des objets Außerseiend». Partant de Meinong, l'auteur nous amène à la thèse de Bolzano selon laquelle «il y a des choses (ou entités) qui ne prétendent pas même à la possibilité », ainsi qu'à sa thèse que la pensée "rien" a un contenu, c'est-à-dire «rien", sans que ce contenu ait un référant.

L'étude des CQR contenant la thèse d'un troisième royaume oriente l'auteur vers la distinction que font Mulligan, Smith et Simons entre truth-maker et truth-bearer ( "véri-facteurs et véri-porteurs »), le truth-maker remplaçant l'Objektiv de Meinong et le Sachverhalt de Stumpf, Reinach, Marty et Husserl. À partir de cette notion, Mulligan, Smith et Simons ont inauguré le Truthmaker Principle. Ce principe implique que pour chaque proposition vraie, il y ait quelque chose dans le monde qui la rende 
vraie. La recherche des CQR à partir de concepts contemporains en tant qu' "outils analytiques" rend ainsi l'ouvrage fort actuel.

L'auteur consacre un chapitre ( "Tropes ou états de choses?») à se demander si Abélard admettrait les «tropes » ou s'il accepterait plutôt la thèse des bundled particulars. Ni l'un ni l'autre, convient l'auteur; Abélard adhérerait plutôt à une Substanceattribute theory des tropes. Et sur la question de la "transférabilité des tropes », il inclinerait vers la thèse de la "sur-venance" (supervenience).

Abélard cède rapidement la parole à Buridan sur la question du «signifié des propositions ". L'auteur présente la thèse de Buridan selon laquelle les propositions «Dieu est Dieu », «Dieu n'est pas Dieu », «Tout Dieu est Dieu », et "Aucun Dieu n'est Dieu ", correspondent au même signifié, mais sous un rapport différent. Encore ici la thèse est mise en relief à travers le concept de truth-maker. La thèse de Buridan est dite « réductionniste", et l'auteur passe donc en revue certaines thèses réductionnistes chez les médiévaux, particulièrement celle d'Oresme voulant que «l'accident n'est rien d'autre que la substance».

Enfin, la réflexion médiévale sur la référence vide, qui commençait vers 1100 avec Abélard, s'achèverait vers 1600 avec Juan Dolz et la théorie non-référentielle d'Enzinas selon lequel les dicta du genre « $p$ signifie que $p$ » n'expriment pas « $p$ signifie que - $p$ » mais que " $p$ signifie - que $p$ ».

Nous affirmions plus haut que l'auteur contribue au projet contemporain de redessiner la carte des antécédents des objets non existants, des propositions et des Sachverhalte vides. Comme nous pouvons le constater, cette carte est vaste, et pourtant l'auteur n'en livre ici que l'esquisse d'une tranche de 500 ans. L'auteur a donc clairement démontré l'ampleur de l'histoire des antécédents de la théorie de l'objet de Meinong, qui ne se résume pas à Grégoire de Rimini, comme le livre de Élie pouvait le laisser entendre, mais s'ouvre et s'étend à tout un complexe de sophismata, maintenu vivant par la méthode scolastique de la disputatio.

FRÉDÉRIC TREMBLAY

Université du Québec à Montréal

\section{Daniel Jacques, La révolution technique : Essai sur le devoir d'humanité, Montréal, Boréal, 2002, 192 pages.}

Empruntant à Martin Heidegger, dont on regrettera que le nom ne soit jamais explicitement mentionné, l'idée que l'essence de la Technique n'est en rien technique ${ }^{10}$, Daniel Jacques s'efforce tout au long de son dernier essai d'identifier les motivations de la technique moderne, faisant de celle-ci, à l'instar de son illustre prédécesseur, une manifestation inédite bien que liée aux époques antérieures de par sa filiation matérielle.

Présentée par D. Jacques comme une révolution, la technique moderne se distingue ainsi des versions précédentes de son évolution en s'apparentant à un projet de réforme ontologique et d'uniformisation socioculturelle de l'humanité.

Tout en demeurant un ensemble de moyens, la Technique se meut, avec l'ère moderne et au gré de son assimilation au Bonheur, en une fin. Lequel Bonheur, bien

10. Martin Heidegger, «La question de la technique », in Essais et Conférences, Vorträge und Aufsätze, 1954, traduit de l'allemand par André Preau, éd. Gallimard, coll. «Tel ». 